\title{
Acute effects of third generation $\beta$-blockers on short-term and beat-to-beat blood pressure variability in sinoaortic-denervated rats
}

\author{
Facundo Martín Bertera ${ }^{1,2,4}$, Julieta Sofía Del Mauro ${ }^{1,4}$, Valeria Lovera ${ }^{1}$, Diego Chiappetta ${ }^{3}$, \\ Ariel Héctor Polizio ${ }^{1}$, Carlos Alberto Taira ${ }^{1,2}$ and Christian Höcht ${ }^{1,2}$
}

An increase in blood pressure variability (BPV) contributes to the development of target organ damage associated with hypertension. Treatment with conventional $\beta$-blockers, such as atenolol, has been associated with an increase in BPV; however, the extrapolation of these results to third generation $\beta$-blockers with pleiotropic effects seems to be inappropriate. The cardiovascular effects of third generation $\beta$-blockers, carvedilol and nebivolol, were assessed in sinoaortic-denervated rats (SAD) and compared with the second generation $\beta$-blocker atenolol and the calcium channel blocker verapamil, with a special focus on short-term BPV. Male SAD rats were acutely treated with carvedilol, nebivolol, atenolol or verapamil at two different doses, and the effects on blood pressure and BPV were recorded. Short-term BPV was assessed by the s.d. of BP recordings. Beat-tobeat BPV was studied using spectral analysis to assess the vascular sympatholytic activity of carvedilol and nebivolol by estimating the effects of these drugs on the ratio of low frequency (LF) to high frequency (HF) BPV (LF/HF ratio). Nebivolol, carvedilol and the calcium channel blocker verapamil significantly attenuated short-term BPV at both doses in SAD animals, and there were no differences between the drugs. Conversely, atenolol did not modify baseline s.d. values at either dose. Carvedilol and nebivolol significantly reduced the LF/HF ratio in SAD rats compared with the effects of atenolol and verapamil, suggesting the ability of the third generation $\beta$-blockers to reduce vascular sympathetic activity. In conclusion, third generation $\beta$-blockers induce a marked reduction in short-term BPV in SAD rats compared to atenolol. Moreover, the ability of carvedilol and nebivolol to reduce short-term BPV in SAD rats is equivalent to that of verapamil, suggesting that these $\beta$-blockers may have an additional beneficial effect through their control of short-term variability to a similar extent to calcium channel blockers.

Hypertension Research (2013) 36, 349-355; doi:10.1038/hr.2012.209; published online 31 January 2013

Keywords: blood pressure variability; calcium channel blockers; sinoaortic denervation; $\beta$-blockers

\section{INTRODUCTION}

Blood pressure is not a constant variable; rather, it shows marked spontaneous oscillations over short-term (minutes to days) and longterm (month) periods. ${ }^{1}$ Increased blood pressure variability (BPV) has been established as a risk factor for the development of target organ damage, not only in hypertensive patients but also in normotensive subjects. ${ }^{1,2}$ A large number of studies have shown that short-term BPV is increased in hypertensive patients compared with age-matched normotensive controls, and the greater oscillation in blood pressure is independently associated with cardiovascular risk. ${ }^{1}$ Indeed, the s.d. of $24 \mathrm{~h}$ blood pressure readings is closely associated with the progression of organ damage. ${ }^{1}$ In addition, recent findings have suggested that elevated BPV also contributes to the development of left ventricular hypertrophy in normotensive subjects and macroalbuminuria in patients with type 2 diabetes mellitus. ${ }^{3,4}$

Considering the contribution of BPV to the development of cardiovascular events, reduction of this parameter may be considered an additional goal for antihypertensive treatment. ${ }^{5}$ Conventional antihypertensive drugs differ in their ability to control excessive fluctuations in blood pressure and therefore have differing clinical benefits. A recent meta-analysis has shown that calcium channel blockers, including dihydropyridines and non-dihydropyridines, are the most effective antihypertensive drugs at reducing BPV. ${ }^{6}$ Conversely, treatment with $\beta$-blockers has been associated with an increase in BPV in hypertensive patients. ${ }^{6}$ Moreover, a subanalysis of the ASCOT-BLPA trial results showed that amlodipine exerts greater protection against cerebrovascular events in hypertensive patients

${ }^{1}$ Department of Pharmacology, University of Buenos Aires, Buenos Aires, Argentina; ${ }^{2}$ Institute of Physiopathology and Clinical Biochemistry, University of Buenos Aires, Buenos Aires, Argentina and ${ }^{3}$ Department of Pharmaceutical Technology, School of Pharmacy and Biochemistry, University of Buenos Aires, Buenos Aires, Argentina ${ }^{4}$ These authors contributed equally to this work.

Correspondence: Dr FM Bertera, Cátedra de Farmacología, Facultad de Farmacia y Bioquímica, Universidad de Buenos Aires, Junín 956, Buenos Aires (C1113AAD), Argentina. E-mail: fbertera@ffyb.uba.ar

Received 10 August 2012; revised 11 September 2012; accepted 14 September 2012; published online 31 January 2013 
than atenolol because of its ability to reduce short-term and longterm BPV. ${ }^{7}$ In another recent report, Mancia et al. ${ }^{8}$ found modestly lower variability of systolic blood pressure values in hypertensive patients treated with lacidipine than in those treated with atenolol. Nevertheless, the extrapolation of these results to third generation $\beta$ blockers, such as carvedilol and nebivolol, seems to be inappropriate, considering $\beta$-blockers greatly differ in their pharmacokinetic and pharmacodynamic properties. ${ }^{9}$ Moreover, a recent systematic review has found that variability in systolic blood pressure is increased more by non-selective $\beta$-blockers than by selective $\beta_{1}$-adrenergic antagonists. ${ }^{10}$

The sinoaortic-denervated $(\mathrm{SAD})$ rat represents an experimental model of increased BPV but normal mean blood pressure. ${ }^{11}$ Previously, SAD rats have been used for the study of acute and chronic effects of antihypertensive drugs on BPV and target organ damage. ${ }^{12-15}$ Nevertheless, to the best our knowledge, effects of third generation $\beta$-blockers on short-term fluctuations of blood pressure have not been studied in this experimental model.

Therefore, the aim of the present work was to compare the effects of atenolol (a second generation $\beta$-blocker), carvedilol (a third generation $\beta$-blocker), nebivolol (a third generation $\beta$-blocker) and verapamil (a calcium channel blocker) on blood pressure and shortterm BPV in SAD rats.

\section{METHODS}

\section{Animals}

Male 3-month-old Wistar rats were used (250-270 g). Animal experiments were performed in accordance with the 'Principles of laboratory animal care' (NIH publication No. 85-3, revised 1985). All efforts were made to minimize animal suffering and to reduce the number of animals used. SAD was performed following the method described by Krieger ${ }^{16}$ under chloral hydrate $\left(250 \mathrm{mg} \mathrm{kg}^{-1}\right.$ i.p.) anesthesia as previously reported. ${ }^{17,18}$ The experiments were carried out 14 days after bilateral SAD. Animals were maintained on a 12 -h light/dark cycle. Rats were kept in a room at $22 \pm 2{ }^{\circ} \mathrm{C}$, and the air was adequately recycled. All animals were fed a standard rodent diet (Asociación Cooperativas Argentinas, Buenos Aires, Argentina) with the following composition (w/w): 20 proteins, 3 fat, 2 fiber, 6 minerals and $69 \%$ starch and vitamin supplements.

\section{Drugs}

Atenolol (Droguerías Saporiti, Buenos Aires Argentina) and verapamil (Droguerías Saporiti, Buenos Aires Argentina) were dissolved in Ringer solution. Owing to their low water solubility, a special formula was prepared for i.v. administration of nebivolol (gift from Laboratorios Raffo, Buenos Aires, Argentina) and carvedilol (Droguerías Saporiti, Buenos Aires Argentina) as previously reported. ${ }^{17,18}$ Ringer solution and the vehicle used in nebivolol and carvedilol solution did not induce significant effects on blood pressure, heart rate (HR) and beat-to-beat and short-term BPV in previous studies of our laboratory. ${ }^{19-21}$ Therefore, to minimize the number of animals used, we did not include vehicle-treated rats in our experimental design.

\section{Experimental design}

Animals were anaesthetized with ether, and the left carotid artery and left femoral vein were cannulated with polyethylene cannulas containing heparinized saline solution $\left(25 \mathrm{U} \mathrm{ml}^{-1}\right)$. Cannulas were tunneled under the skin and externalized at the back of the neck. Experiments were performed in freely moving animals $24 \mathrm{~h}$ after cannula placement. A recovery period of $24 \mathrm{~h}$ has been found to be adequate for evaluation of drug effects on BPV in conscious rats.

On the day of the experiment, the arterial cannula was connected to a Spectramed P23XL pressure transducer (Spectramed, Oxnard, CA, USA) coupled to a Grass 79D polygraph (Grass Instrument, Quincy, MA, USA). The polygraph was connected to a digital converter adaptor unit (Polyview, PVA 1, Grass-Astro Med, West Warwick, RI, USA), and recordings were stored and analyzed with a software program (Polyview 2.3 Astro-Med). Basal mean arterial pressure (MAP) and HR were estimated during a 60-min interval before drug administration. MAP was calculated as the sum of the diastolic pressure and one-third of the pulse pressure. HR was estimated tachographically by counting the pulsatile waves of the arterial pressure recording.

Atenolol $\left(0.3\right.$ or $\left.3 \mathrm{mg} \mathrm{kg}^{-1}\right)$, nebivolol $\left(0.3\right.$ or $\left.3 \mathrm{mg} \mathrm{kg}^{-1}\right)$, carvedilol $(0.1$ or $1 \mathrm{mg} \mathrm{kg}^{-1}$ ) or verapamil ( 0.3 or $\left.3 \mathrm{mg} \mathrm{kg}^{-1}\right)$ ( $n=6$ for each group) was injected i.v. over $30 \mathrm{~s}$. After drug administration, MAP and HR were continuously recorded for $3 \mathrm{~h}$, and the blood pressure recording was stored for further analysis. To reduce the effects attributable to circadian alterations, all experiments were conducted between 1300 hours and 1900 hours. Drug dosages were selected to achieve an equivalent maximal chronotropic response to the different $\beta$-blockers evaluated in the study.

\section{Estimation of BPV}

BPV was continuously estimated by determination of s.d. and spectral analysis of 3 min periods of blood pressure recordings obtained at baseline and at regular times after drug administration, when the quality of the arterial blood pressure signal was visually considered to be satisfactory. As previously reported, ${ }^{19,20,22,23}$ spectral analysis of the data was performed using the Fast Fourier Transform algorithm with a Hamming window (Polyview 2.3 AstroMed). The total power spectral density, an index of global variability, was calculated by integrating the power spectra over the frequency range $(0.1-$ $2.5 \mathrm{~Hz})$. Spectral densities in the total power spectral density in the very low frequency (VLF) range $(0.1-0.2 \mathrm{~Hz})$, the low frequency $(\mathrm{LF})$ range $(0.2-0.7 \mathrm{~Hz})$ and the high frequency (HF) range $(0.7-2.5 \mathrm{~Hz})$ were calculated from baseline recordings and after drug administration as previously reported. ${ }^{19,20,22}$ Although LF variability is affected by sympathetic modulation of vascular tone, we used the LF/HF ratio as an index of vascular sympathetic activity. The normalization procedure and estimation of the LF/HF ratio tends to minimize the effect of the changes in total power on the absolute values of LF variability. 23,24

\section{Statistical Analysis}

Normal distribution of the data and the variables of the study were verified using the Kolmogorov-Smirnov test. Data are expressed as the means \pm s.e.m. Drug effects on MAP, HR, s.d. and LF/HF ratio were compared by means of one- or two-way analyses of variance and Bonferroni's post hoc test. Statistical tests were performed using GraphPad Prism version 5.02 for Windows (GraphPad Software, San Diego, California, CA, USA). Statistical significance was defined as $P<0.05$.

\section{RESULTS}

After 14 days of sinoaortic denervation, SAD rats showed an increase in the s.d. of blood pressure recordings $(7.9 \pm 0.5 \mathrm{~mm} \mathrm{Hg}, n=24)$ and normal baseline MAP values $(112 \pm 2, n=24)$ compared with historical data from sham-operated rats (s.d.: $4.1 \pm 0.7 \mathrm{~mm} \mathrm{Hg}$, $n=12$; MAP: $109 \pm 3 \mathrm{~mm} \mathrm{Hg}, n=12$ ) obtained in our laboratory.

Figure 1 shows the effects of low and high doses of carvedilol, nebivolol, atenolol and verapamil on MAP in SAD rats. The low dose of the antihypertensive drugs induced only minor changes in baseline blood pressure values (Figure 1a). Statistical analysis of the results showed a greater hypotensive response to $0.1 \mathrm{mg} \mathrm{kg}^{-1}$ carvedilol than to $0.3 \mathrm{mg} \mathrm{kg}^{-1}$ nebivolol. After i.v. application of the high dose, nebivolol, verapamil and carvedilol induced a greater blood pressure lowering effect than that of $3 \mathrm{mg} \mathrm{kg}^{-1}$ atenolol (Figure $1 \mathrm{~b}$ ). Moreover, two-way analyses of variance analysis showed a greater MAP reduction after acute administration of carvedilol and verapamil than nebivolol (Figure 1b). Nevertheless, nebivolol showed a delayed effect on blood pressure, whereas carvedilol and verapamil induced a fast hypotensive response with rapid recovery of MAP to baseline values.

All antihypertensive drugs induced a dose-dependent reduction in HR in SAD rats (Figure 2). After administration of the low dose, the chronotropic response to different b-blockers, including atenolol, 

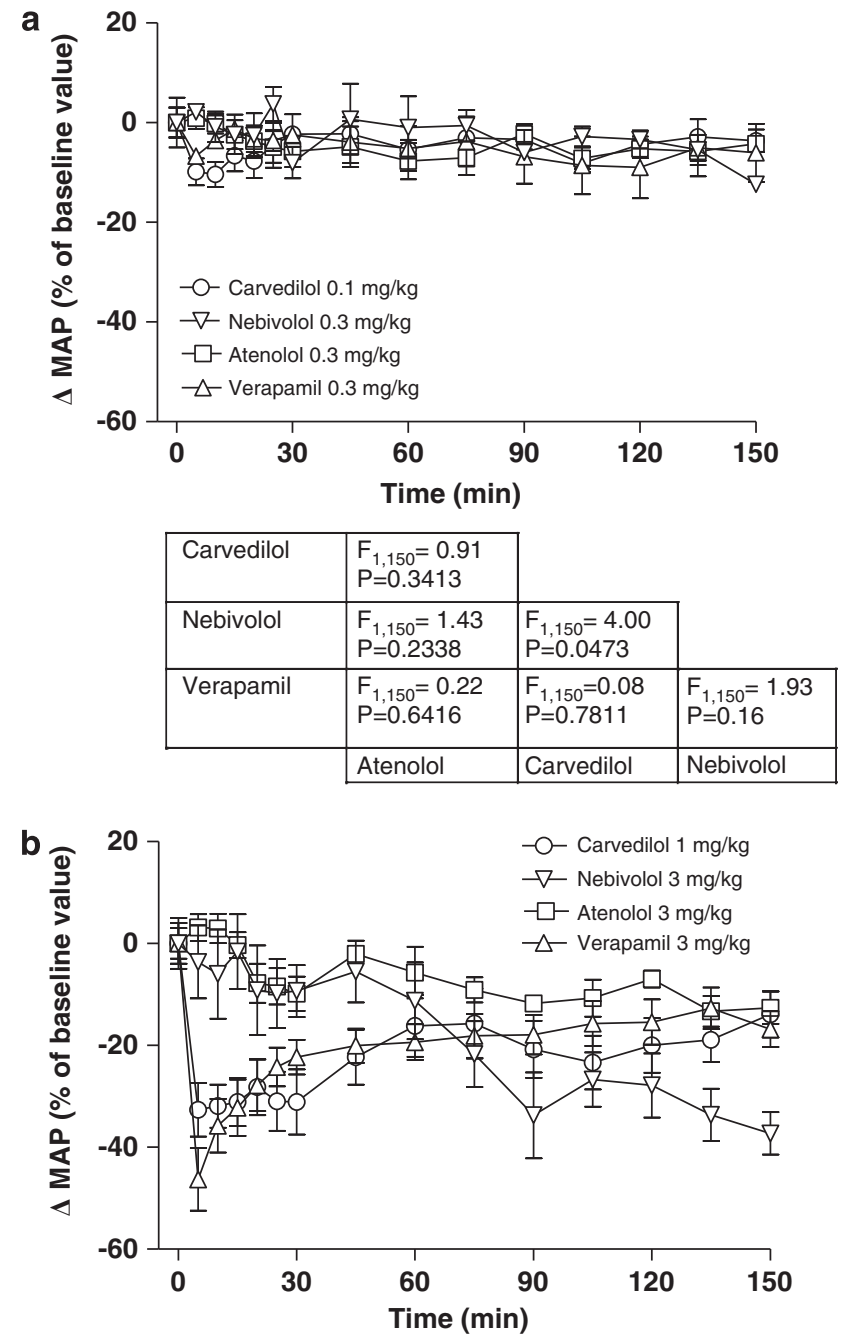

\begin{tabular}{|l|l|l|l|}
\hline Carvedilol & $\mathrm{F}_{1,150}=102.3$ & \multicolumn{1}{|}{} \\
& $\mathrm{P}<0.0001$ & \multicolumn{2}{|c}{} \\
\cline { 1 - 2 } Nebivolol & $\mathrm{F}_{1,150}=25.98$ & $\mathrm{~F}_{1,150}=9.11$ & \\
& $\mathrm{P}<0.0001$ & $\mathrm{P}=0.003$ & \\
\hline Verapamil & $\mathrm{F}_{1,150}=124.0$ & $\mathrm{~F}_{1,150}=0.21$ & $\mathrm{~F}_{1,150}=8.12$ \\
& $\mathrm{P}<0.0001$ & $\mathrm{P}=0.65$ & $\mathrm{P}=0.005$ \\
\hline & Atenolol & Carvedilol & Nebivolol \\
\cline { 2 - 3 } & & &
\end{tabular}

Figure 1 Time course of changes in mean arterial pressure ( $\triangle M A P, \%$ of baseline values), after i.v. administration of low (a) or high (b) doses of carvedilol (circles), nebivolol (inverse triangle), atenolol (square) or verapamil (triangle) to sinoaortic-denervated (SAD) rats. Each point shows the mean \pm s.e.m. of six rats. The table shows the statistical parameters of drug comparisons after two-way analysis of variance (ANOVA).

nebivolol and carvedilol, was not different from the response to verapamil (Figure $2 \mathrm{a}$ ). In addition, atenolol induced a slightly greater negative chronotropic response than that induced by carvedilol. Conversely, no differences were found in the bradycardic response to $0.1 \mathrm{mg} \mathrm{kg}^{-1}$ carvedilol and $0.3 \mathrm{mg} \mathrm{kg}^{-1}$ nebivolol (Figure $2 \mathrm{a}$ ).

After i.v. administration of the high dose, the maximal bradycardic effect of verapamil was enhanced with respect to $3 \mathrm{mg} \mathrm{kg}^{-1}$ nebivolol, $1 \mathrm{mg} \mathrm{kg}^{-1}$ carvedilol and $3 \mathrm{mg} \mathrm{kg}^{-1}$ atenolol. No differences were found in the maximal chronotropic response among the $\beta$-blockers, although the effect on HR was prolonged in SAD animals treated with

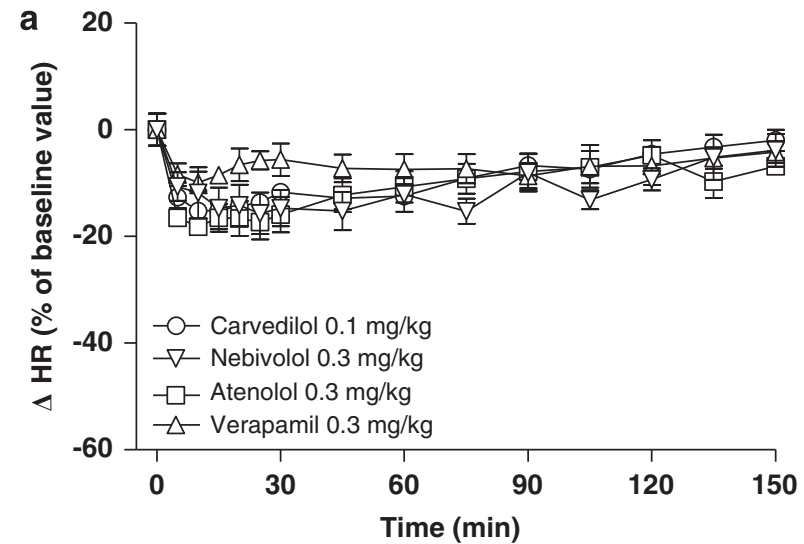

\begin{tabular}{|c|c|c|c|}
\hline Carvedilol & $\begin{array}{l}F_{1,150}=0.56 \\
P=0.8945\end{array}$ & & \\
\hline Nebivolol & $\begin{array}{l}F_{1,150}=0.81 \\
P=0.6553\end{array}$ & $\begin{array}{l}F_{1,150}=0.53 \\
P=0.9090\end{array}$ & \\
\hline \multirow[t]{2}{*}{ Verapamil } & $\begin{array}{l}F_{1,150}=1.31 \\
P=0.2075\end{array}$ & $\begin{array}{l}F_{1,150}=1.61 \\
P=0.0824\end{array}$ & $\begin{array}{l}F_{1,150}=0.76 \\
P=0.71450\end{array}$ \\
\hline & Atenolol & Carvedilol & Nebivolol \\
\hline
\end{tabular}

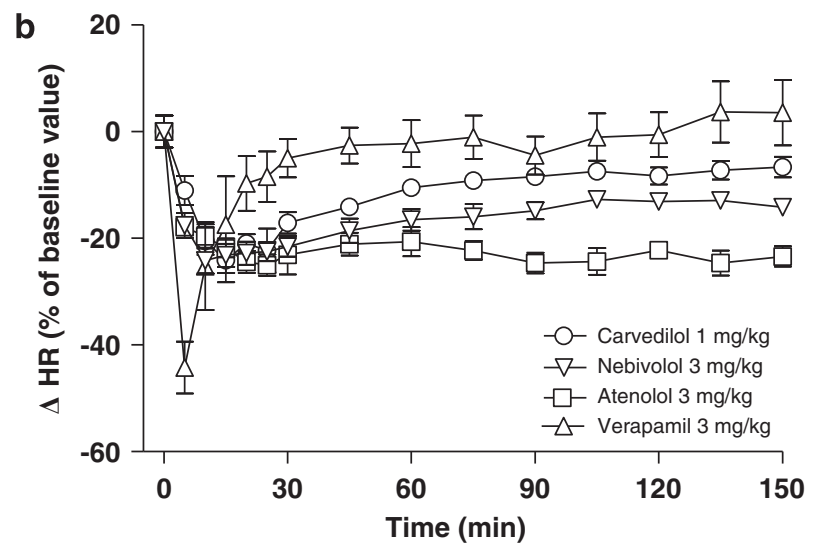

\begin{tabular}{|c|c|c|c|}
\hline Carvedilol & $\begin{array}{l}\mathrm{F}_{1,150}=106.2 \\
\mathrm{P}<0.0001\end{array}$ & & \\
\hline Nebivolol & $\begin{array}{l}F_{1,150}=31.18 \\
P<0.0001\end{array}$ & $\begin{array}{l}F_{1,150}=29.11 \\
P=0.0001\end{array}$ & \\
\hline \multirow[t]{2}{*}{ Verapamil } & $\begin{array}{l}\mathrm{F}_{1,150}=82.41 \\
\mathrm{P}<0.0001\end{array}$ & $\begin{array}{l}F_{1,150}=10.98 \\
P=0.0012\end{array}$ & $\begin{array}{l}F_{1,150}=39.27 \\
P=0.0001\end{array}$ \\
\hline & Atenolol & Carvedilol & Nebivolol \\
\hline
\end{tabular}

Figure 2 Time course of changes in heart rate ( $\Delta \mathrm{HR}, \%$ of baseline values), after i.v. administration of low (a) or high (b) doses of carvedilol (circles), nebivolol (inverse triangle), atenolol (square) or verapamil (triangle) to sinoaortic-denervated (SAD) rats. Each point shows the mean \pm s.e.m. of six rats. The table shows the statistical parameters of drug comparisons after two-way analysis of variance (ANOVA).

$3 \mathrm{mg} \mathrm{kg}^{-1}$ atenolol compared with those treated with carvedilol, nebivolol and verapamil.

The effects of the drugs on short-term BPV, expressed as the s.d. of blood pressure recordings, in SAD rats are depicted in Figure 3. After administration of the low dose, the third generation $\beta$-blockers nebivolol and carvedilol and the calcium channel blocker verapamil significantly attenuated short-term BPV in SAD animals, and no differences were observed between the drugs (Figure 3a). Conversely, $0.3 \mathrm{mg} \mathrm{kg}^{-1}$ atenolol did not modify baseline s.d. values throughout the entire experiment (Figure 3a). 


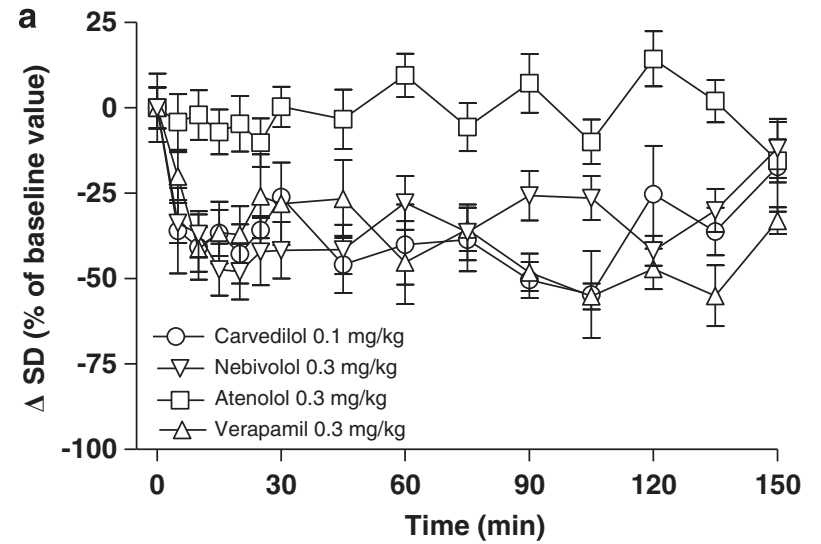

\begin{tabular}{|l|l|l|l|}
\hline Carvedilol & $\begin{array}{l}\mathrm{F}_{1,150}=60.6 \\
\mathrm{P}<0.0001\end{array}$ & \multicolumn{2}{|l}{} \\
\cline { 1 - 2 } Nebivolol & $\mathrm{F}_{1,150}=53.53$ & $\mathrm{~F}_{1,150}=0.46$ & \multicolumn{1}{|l}{} \\
& $\mathrm{P}<0.0001$ & $\mathrm{P}=0.5047$ & \\
\hline Verapamil & $\mathrm{F}_{1,150}=139.3$ & $\mathrm{~F}_{1,150}=0.03$ & $\mathrm{~F}_{1,150}=0.81$ \\
& $\mathrm{P}<0.0001$ & $\mathrm{P}=0.8546$ & $\mathrm{P}=0.3695$ \\
& Atenolol & Carvedilol & Nebivolol \\
\cline { 2 - 3 } & & & \\
\hline
\end{tabular}

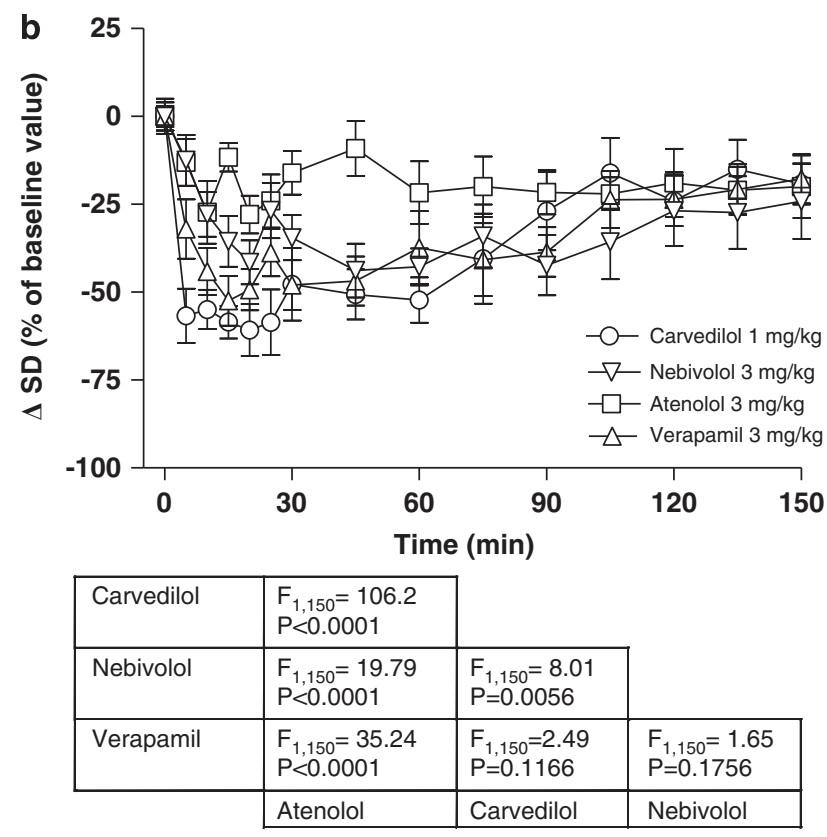

Figure 3 Changes in short-term blood pressure variability, expressed as \% of baseline s.d. value, after i.v. administration of low (a) or high (b) doses of carvedilol (circles), nebivolol (inverse triangle), atenolol (square) or verapamil (triangle) to sinoaortic-denervated (SAD) rats. Each point shows the mean \pm s.e.m. of six rats. The table shows the statistical parameters of drug comparison after two-way analysis of variance (ANOVA).

At the high dose level, atenolol $\left(3 \mathrm{mg} \mathrm{kg}^{-1}\right)$ exerted negligible effects on short-term BPV, whereas carvedilol, nebivolol and verapamil dramatically reduced the s.d. of blood pressure recordings in SAD rats (Figure $3 \mathrm{~b}$ ). Thus, the drug effects on short-term BPV were significantly greater after i.v. administration of $1 \mathrm{mg} \mathrm{kg}^{-1}$ carvedilol, $3 \mathrm{mg} \mathrm{kg}^{-1}$ nebivolol or $3 \mathrm{mg} \mathrm{kg}^{-1}$ verapamil than $3 \mathrm{mg} \mathrm{kg}^{-1}$ atenolol (Figure 3b). Carvedilol induced a greater reduction in s.d. values in $\mathrm{SAD}$ rats than $3 \mathrm{mg} \mathrm{kg}^{-1}$ nebivolol. No significant differences were found in the effects on BPV between nebivolol or carvedilol and $3 \mathrm{mg} \mathrm{kg}^{-1}$ verapamil (Figure $3 \mathrm{~b}$ ).

\section{Drug effects on beat-to-beat BPV in SAD rats}

The effects of the drugs on beat-to-beat BPV obtained by spectral analysis of blood pressure recording in SAD rats are shown in Table 1. Carvedilol, nebivolol and verapamil, but not atenolol, reduced the total power spectral density, an index of global variability, after administration of a high dose to SAD rats (Table 1). Moreover, a low dose of carvedilol was more effective than atenolol and verapamil at reducing beat-to-beat global variability of blood pressure. After acute i.v. administration of the low dose, only carvedilol significantly reduced VLF BPV with respect to $0.3 \mathrm{mg} \mathrm{kg}^{-1}$ atenolol (Table 1). At the high dose, carvedilol, nebivolol and verapamil clearly attenuated VLF BPV in SAD animals compared with atenolol but without significant differences between all three drugs.

At the LF domain of BPV, $0.1 \mathrm{mg} \mathrm{kg}^{-1}$ carvedilol was more effective at controlling LF BPV than $0.3 \mathrm{mg} \mathrm{kg}^{-1}$ atenolol, $0.3 \mathrm{mg} \mathrm{kg}^{-1}$ nebivolol and $0.3 \mathrm{mg} \mathrm{kg}^{-1}$ verapamil (Table 1 ). Conversely, at the high dose, carvedilol and verapamil showed more attenuation of LF BPV in SAD animals than atenolol and nebivolol. In addition, $3 \mathrm{mg} \mathrm{kg}^{-1}$ nebivolol induced a greater reduction of BPV in the LF domain than $3 \mathrm{mg} \mathrm{kg}^{-1}$ atenolol (Table 1).

In addition, all antihypertensive drugs evaluated in the study showed negligible effects on HF BPV after acute administration of the low dose (Table 1). Conversely, at the high doses, carvedilol, nebivolol and verapamil significantly reduced HF variability in blood pressure recordings compared with the variability observed with atenolol. In addition, carvedilol and verapamil exhibited greater efficacy in reducing HF BPV than nebivolol (Table 1).

The effects of the drugs on the LF/HF ratio, a marker of vascular sympathetic activity, after acute i.v. administration to SAD animals are shown in Figure 4. After i.v. application of the low dose, carvedilol and nebivolol induced a greater attenuation of the LF/HF ratio than atenolol and verapamil (Figure 4a). In addition, the LF/HF ratio was markedly reduced after $0.1 \mathrm{mg} \mathrm{kg}^{-1}$ carvedilol administration in comparison with $0.3 \mathrm{mg} \mathrm{kg}^{-1}$ nebivolol (Figure 4a). Similar findings were found in SAD rats after administration of the high dose of the antihypertensive drugs applied in the study. Both carvedilol and nebivolol significantly reduced the LF/HF ratio in SAD rats compared with the ratio in atenolol or verapamil-treated rats (Figure $4 \mathrm{~b}$ ). No differences were found between the effects of carvedilol and nebivolol on the LF/HF ratio after the administration of high doses (Figure 4b).

\section{DISCUSSION}

Considering the contribution of the BPV to cardiovascular risk and target organ damage in hypertension and the fact that the lower protective effect of traditional $\beta$-blockers has been attributed to their inability to attenuate increased oscillations of blood pressure, the present study was designed to compare the acute effects of second generation and third generation $\beta$-blockers and calcium channel blockers on short-term BPV in SAD animals.

The SAD rat, a high-BPV model, represents the gold standard for the study of the physiology, pathology and pharmacology related to BPV. Studies in SAD rats have demonstrated the development of target organ damage, such as myocardial damage, renal lesions and vascular remodeling after 4 weeks of denervation. ${ }^{11}$ Different mechanisms have been proposed to be involved in the target organ damage associated with high BPV, including hemodynamic effects, endothelial dysfunction and activation of the renin-angiotensin system and the sympathetic nervous system. ${ }^{11}$ Although 
Table 1 Change in beat-to-beat blood pressure variability in the, VLF, LF and HF domain (expressed as \% of baseline values) after i.v. administration of low and high dose of carvedilol, nebivolol, atenolol and verapamil in SAD rats

\begin{tabular}{|c|c|c|c|c|c|c|c|c|}
\hline & \multicolumn{2}{|c|}{ Atenolol } & \multicolumn{2}{|c|}{ Carvedilol } & \multicolumn{2}{|c|}{ Nebivolol } & \multicolumn{2}{|c|}{ Verapamil } \\
\hline & $0.3 \mathrm{mg} \mathrm{kg}^{-1}$ & $3 \mathrm{mg} \mathrm{kg}^{-1}$ & $0.1 \mathrm{mg} \mathrm{kg}^{-1}$ & $1 \mathrm{mg} \mathrm{kg}^{-1}$ & $0.3 \mathrm{mg} \mathrm{kg}^{-1}$ & $3 \mathrm{mg} \mathrm{kg}^{-1}$ & $0.3 \mathrm{mg} \mathrm{kg}^{-1}$ & $3 \mathrm{mg} \mathrm{kg}^{-1}$ \\
\hline Total power density & $-3.8 \pm 5.4$ & $8.6 \pm 5.8$ & $-39.7 \pm 8.6^{*, \#}$ & $-56.3 \pm 5.9 *, \S$ & $-21.4 \pm 3.3$ & $-28.9 \pm 5.6^{*}$ & $-11.3 \pm 2.7$ & $-58.7 \pm 5.8^{\star, \S}$ \\
\hline VLF & $3.9 \pm 3.5$ & $11.8 \pm 2.9$ & $-41.4 \pm 8.6^{*}$ & $-54.2 \pm 5.1^{*}$ & $-17.52 \pm 10.3$ & $-35.9 \pm 8.8^{*}$ & $-14.2 \pm 3.6$ & $-57.9 \pm 6.3^{*}$ \\
\hline LF & $-1.3 \pm 2$ & $3.3 \pm 9.5$ & $-39.9 \pm 8.4 *,, \#$ & $-60.2 \pm 4.9 *, \S$ & $-11.9 \pm 4.9$ & $-29.1 \pm 6.5^{*}$ & $-13.2 \pm 3.5$ & $-59.1 \pm 5^{*, \S}$ \\
\hline $\mathrm{HF}$ & $-1.3 \pm 2.7$ & $8.8 \pm 6.2$ & $-26.1 \pm 9.1$ & $-52.7 \pm 7^{*, \S}$ & $-7.6 \pm 8$ & $-22.9 \pm 7.2^{*}$ & $-11.1 \pm 1.9$ & $-52.2 \pm 5.5^{*, \S}$ \\
\hline
\end{tabular}

Abbreviations: HF, high frequency; LF, low frequency; SAD, sinoaortic denervated; VLF, very low frequency.

Each bar shows the mean \pm s.e.m. of six rats. ${ }^{\star} P<0.05$ vs. atenolol; ${ }^{\circledR} P<0.05$ vs. nebivolol; $\# P<0.05$ vs. verapamil by one-way ANOVA followed by Bonferroni's post hoc- test.
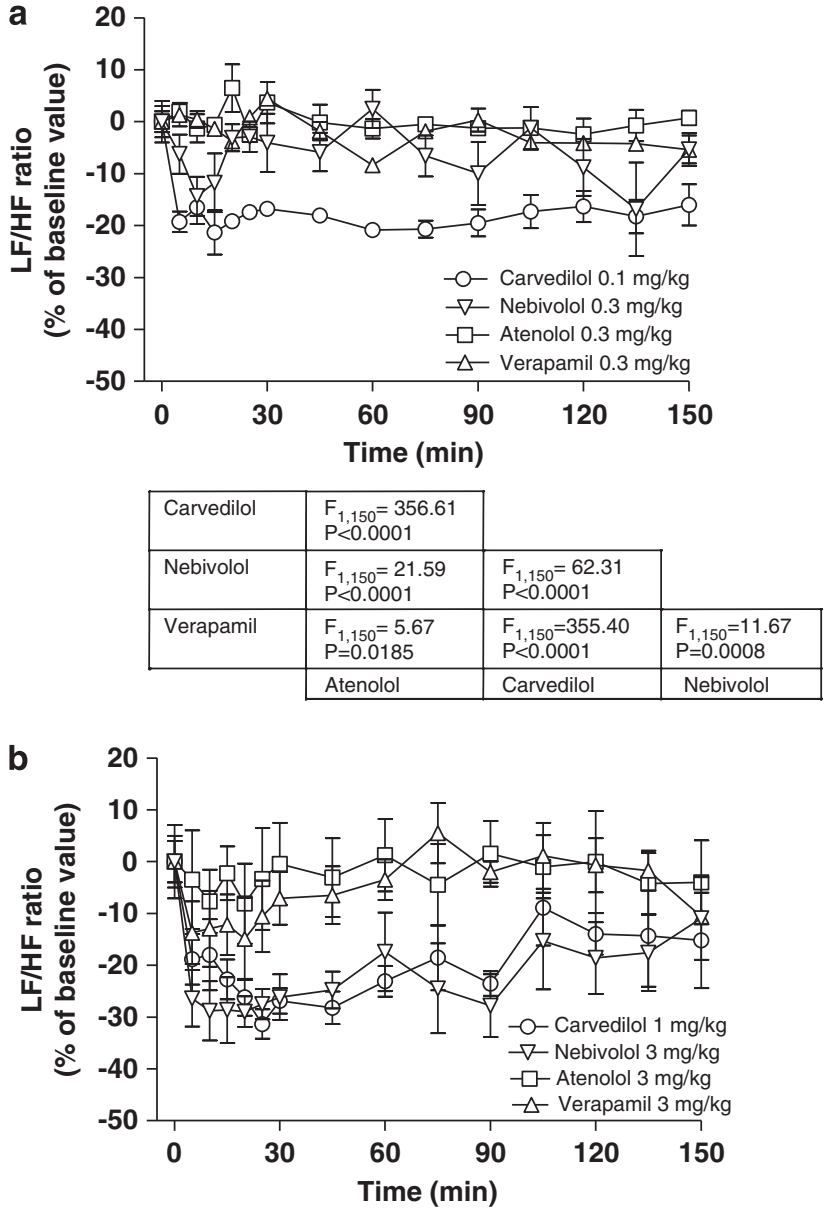

\begin{tabular}{|l|l|l|l|}
\hline Carvedilol & $\mathrm{F}_{1,150}=48.80$ & \multicolumn{1}{|c}{} \\
& $\mathrm{P}<0.0001$ & \multicolumn{1}{|c}{} \\
\cline { 1 - 2 } Nebivolol & $\mathrm{F}_{1,150}=55,35$ & $\mathrm{~F}_{1,150}=1.06$ & \\
& $\mathrm{P}<0.0001$ & $\mathrm{P}=0.3055$ & \\
\hline Verapamil & $\mathrm{F}_{1,150}=1,99$ & $\mathrm{~F}_{1,150}=44.59$ & $\mathrm{~F}_{1,150}=51.02$ \\
& $\mathrm{P}_{1,0.16}$ & $\mathrm{P}=0.0001$ & $\mathrm{P}=0.0001$ \\
\hline & Atenolol & Carvedilol & Nebivolol \\
\cline { 2 - 3 } & &
\end{tabular}

Figure 4 Time course of changes in normalized low frequency (LF) variability (LF/HF ratio), expressed as \% of baseline values, after i.v. administration of low (a) or high (b) doses of carvedilol (circles), nebivolol (inverse triangle), atenolol (square) or verapamil (triangle) to sinoaorticdenervated $(S A D)$ rats. Each point shows the mean \pm s.e.m. of six rats. The table shows the statistical parameters of drug comparison after two-way analysis of variance (ANOVA).

sympathetic overactivity contributes to BPV early in the first $24 \mathrm{~h}$ after sinoaortic denervation, its activity returns to the normal level In the chronic stage of SAD rats. $^{25}$
There is evidence that antihypertensive drugs are effective at the attenuation of short-term BPV in SAD rats after acute administration. Wang et al. ${ }^{12}$ demonstrated that calcium channel blockers (nifedipine, nitrendipine and amlodipine) and drugs acting on the sympathetic nervous system (clonidine, prazosin and atenolol) significantly decreased BPV in SAD rats. Conversely, telmisartan, hydrochlorothiazide and captopril were found not to modify BPV in SAD rats after acute administration. ${ }^{12}$ Nevertheless, to the best of the our knowledge, the effects of the acute administration of third generation $\beta$-blockers, carvedilol and nebivolol, and the calcium channel blocker verapamil have not previously been studied in this experimental model of high BPV.

In the present study, we compared the acute effects of a low and a high dose of atenolol, carvedilol, nebivolol and verapamil on shortterm BPV, beat-to-beat BPV, MAP and HR in SAD rats. As the BPV increases with baseline MAP, it is important to distinguish whether the beneficial effects of antihypertensive drugs on BPV are independent of the hypotensive response. At a low dose, none of the atenolol, carvedilol, nebivolol or verapamil treatments induced a significant reduction in MAP in SAD rats. However, carvedilol, nebivolol and verapamil, but not atenolol, significantly attenuated high short-term BPV in this experimental model, suggesting that the efficacy of third generation $\beta$-blockers and calcium channel blockers at reducing excessive BPV is not directly related to their hypotensive action. Our results are in agreement with previous findings by Van Vliet and Chafe $^{26}$ that showed that amlodipine can reduce MAP variability independently of changes in the mean blood pressure level in baroreceptor-denervated rats. In addition, comparison of the low dose drug effects on short-term BPV showed that the third generation $\beta$-blockers are more effective than atenolol at the reduction of the s.d. of blood pressure recordings in SAD rats. Moreover, subdepressor doses of carvedilol and nebivolol showed an ability to attenuate shortterm BPV that was comparable to the effects of the calcium channel blocker verapamil.

At a high dose, nebivolol, carvedilol and verapamil exerted greater hypotensive activity in SAD rats than the activity of atenolol. In addition, the maximal hypotensive response to carvedilol, verapamil and nebivolol was similar, although nebivolol induced a delayed blood pressure lowering action compared with the fast response to i.v. administration of carvedilol and verapamil. The pattern of blood pressure changes described in the present study agrees with previous results of pharmacokinetic-pharmacodynamic studies in other experimental models. ${ }^{19-21}$ Both carvedilol and verapamil have been found to produce fast and short-acting hypotensive responses in experimental models of hypertension. ${ }^{20,21}$ Sudden drops in MAP detected after carvedilol and verapamil can be observed only after i.v. administration and can result in reflex sympathetic activation. However, reflex sympathetic activation due to the hypotension 
induced by carvedilol and verapamil is blunted in SAD rats as a result of a marked reduction in baroreflex sensitivity. ${ }^{27}$ Nevertheless, the possibility of sympathetic overactivity after i.v. administration of high doses of carvedilol and verapamil must be considered in subjects with normal baroreflex sensitivity. Conversely, nebivolol exerted a long lasting and delayed hypotensive response after acute i.v. administration in normotensive and spontaneously hypertensive rats. ${ }^{19}$ Several mechanisms could contribute to the sustained hypotensive efficacy of nebivolol, including its slow dissociation from its receptor and the generation of active metabolites. ${ }^{28}$ Meier et al. ${ }^{29}$ have found a long-lasting hypotensive response to acute nebivolol administration in SHR that has been attributed to its active metabolites.

Comparison of the drugs' effects on BPV after administration with the high dose again demonstrated a greater efficacy of the third generation $\beta$-blockers carvedilol and nebivolol in controlling shortterm oscillations of blood pressure than the efficacy of atenolol. Moreover, the vasodilatory $\beta$-blockers and verapamil had a similar ability to attenuate BPV, reaffirming that the pleiotropic effects of third generation $\beta$-blockers greatly are beneficial not only in their hypotensive action but also in the attenuation of short-term BPV. Although the results of our study must be interpreted with caution, considering the short-term nature of the study, the findings suggest that the inefficacy of traditional $\beta$-blockers, such as atenolol, to reduce short-term BPV cannot be extrapolated to third generation $\beta$-blockers with vasodilatory properties. Although the mechanisms involved in the beneficial effects of third generation $\beta$-blockers on short-term BPV in SAD rats are unknown, enhancement of endothelial function mediated by carvedilol and nebivolol could contribute to their ability to reduce blood pressure oscillations. In this context, Rocha and Bendhack ${ }^{30}$ have found the presence of spontaneous oscillatory contractions in aortas isolated from SAD rats, a mechanism that could contribute to blood pressure lability in this experimental group. Spontaneous oscillatory contractions in SAD rats were increased after the removal of the aortic endothelium, suggesting the involvement of endothelium-derived factors in the prevention of BPV in SAD rats. ${ }^{30}$ Preclinical and clinical studies have demonstrated that both carvedilol and nebivolol are capable of enhancing endothelial function in hypertensive patients. ${ }^{31,32}$ Taken together, the enhancement of endothelial function by carvedilol and nebivolol may be involved in the observed attenuation of short-term BPV in SAD rats after the administration of low or high doses. In addition, Rocha and Bendhack $^{30}$ have found that spontaneous oscillatory contractions in aortas from SAD rats are prevented by low concentrations of verapamil, suggesting the participation of calcium channels in BPV in this experimental model.

Overall, BPV is composed of two variables: the cyclic components detected by spectral analysis of blood pressure recordings and the erratic variability. Although the cyclic components of BPV do not show a close relationship with target organ damage, identification of the frequency components of BPV by power spectral analysis can potentially provide information about the mechanisms involved in blood pressure regulation. ${ }^{33}$ In this context, the renin-angiotensin system peptides, catecholamines, endothelial-derived NO and myogenic vascular function affect BPV at VLF. ${ }^{33}$ Conversely, LF variability is modulated by sympathetic modulation of vascular tone and endothelial-derived NO in rats. ${ }^{33}$ Variability in the HF domain is mainly influenced by changes in cardiac output. ${ }^{34}$ In addition, normalized LF (LF/HF ratio) has been validated as a marker of sympathetic vascular activity in preclinical and clinical studies. ${ }^{24,35}$
Acute administration of carvedilol, nebivolol and verapamil induced a reduction of all components of cyclic BPV, including VLF, LF and HF variability. Conversely, atenolol did not modify beatto-beat BPV. In addition, carvedilol showed a greater ability to reduce VLF and LF variability, considering the fact that its effects on beat-tobeat BPV were evident at a low dose. The lack of effect of atenolol on cyclic components of BPV could be explained by the fact that this cardioselective $\beta$-blocker did not affect the neurohumoral control of blood pressure or vascular sympathetic activity. Consistent with our findings, Just et al. ${ }^{36}$ have found that atenolol administration in mice does not significantly change different domains of beat-to-beat BPV, despite its strong effects on HR.

The ability of carvedilol to attenuate beat-to-beat BPV in the VLF, LF and HF domains is most likely a consequence of its antioxidant and vascular sympatholytic activity and the reduction of cardiac output instability. Similar findings have been made in our laboratory in different models of hypertension, including spontaneously hypertensive rats, fructose-fed rats and L-NAME hypertensive animals. ${ }^{20,22,37}$ In all experimental subjects, acute i.v. application of carvedilol greatly reduced BPV in the three frequency domains. Moreover, the vascular sympatholytic activity of carvedilol is also evidenced by the reduction of the LF/HF ratio, a validated index of vascular sympathetic activity.

Nebivolol also induced a marked attenuation of VLF, LF and HF BPV in SAD rats after application of the high dose. Similar findings have recently been made in our laboratory after acute administration of nebivolol to spontaneously hypertensive and normotensive rats. ${ }^{19}$ The effects of this third generation $\beta$-blocker on beat-to-beat BPV, as assessed by spectral analysis, could be explained by its ability to enhance endothelial-derived NO activity. In addition, analysis of the drug's effects on the LF/HF ratio also showed that nebivolol significantly attenuated the ratio after i.v. administration. Several previous studies have described the sympatholytic actions of nebivolol, which are partially related to the increase in NO bioavailability. ${ }^{38,39}$ Sacco et al. $^{39}$ found that central application of nebivolol can acutely increase brain NO release, thereby reducing the activity of the sympathetic nervous system.

Finally, in our study, i.v. administration of verapamil also induced a marked attenuation of beat-to-beat BPV in the VLF, LF and HF domains. To the best of our knowledge, the effects of verapamil On beat-to-beat BPV, as measured by spectral analysis recordings, have not previously been studied in laboratory animals. Nevertheless, other authors have found that nifedipine, a dihydropiridine calcium channel blocker, reduces VLF and LF BPV in conscious normotensive Wistar-Kyoto rats. ${ }^{33}$ These effects have been attributed to the ability of the calcium channel blocker to prevent the vascular myogenic response, as well as to the reduction of $\alpha_{1}$-adrenoceptor-mediated vasoconstriction via blockade of store-operated $\mathrm{Ca}^{2+}$ channels. ${ }^{33}$

In conclusion, comparison of the effects of second- and thirdgeneration $\beta$-blockers and the calcium channel blocker verapamil on $\mathrm{BPV}$ in SAD rats demonstrated that nebivolol and carvedilol show improved cardiovascular properties compared with atenolol treatment, inducing a marked reduction in short-term BPV in SAD rats. Moreover, the ability of carvedilol and nebivolol to reduce short-term $\mathrm{BPV}$ in SAD rats is equivalent to that of verapamil, suggesting that these $\beta$-blockers provide beneficial cardiovascular effects by controlling short-term variability to a similar extent to calcium channel blockers. Our results suggest that the ineffectiveness of atenolol to control excessive fluctuations in blood pressure must not be extrapolated to $\beta$-blockers with pleiotropic effects, such as carvedilol and nebivolol. In addition, as expected, carvedilol and nebivolol showed 
greater hypotensive activity than atenolol in SAD rats, which is associated with their ability to enhance endothelial-derived NO activity and to interfere with vascular sympathetic tone. The results of the present study provide new insight into the effects of these drugs on short-term BPV and provide motivation for the development of long-term studies to elucidate whether the beneficial effects of carvedilol and nebivolol on BPV continue during long-term administration and whether these treatments result in a greater target organ protection than does atenolol.

\section{CONFLICT OF INTEREST}

The authors declare no conflict of interest.

\section{ACKNOWLEDGEMENTS}

This work was supported by grants from Secretaría de Ciencia y Técnica, Universidad de Buenos Aires, Argentina and from the Agencia Nacional de Promoción Científica (Préstamo BID PICT 00994). Diego Chiappetta, Ariel H Polizio and Carlos A Taira are Career Investigators from CONICET, Argentina.

1 Grassi G, Bombelli M, Brambilla G, Trevano FQ, Dell'oro R, Mancia G. Total cardiovascular risk, blood pressure variability and adrenergic overdrive in hypertension: evidence, mechanisms and clinical implications. Curr Hypertens Rep 2012; 14 333-338.

2 Parati G. Blood pressure variability: its measurement and significance in hypertension. $J$ Hypertens 2005; 23 (suppl 1): S19-S25.

3 Schutte AE, Schutte R, Huisman HW, van Rooyen JM, Fourie CM, Malan NT, Malan L. Blood pressure variability is significantly associated with ECG left ventricular mass in normotensive Africans: the SABPA Study. Hypertens Res 2011; 34: 1127-1134.

4 Ushigome E, Fukui M, Hamaguchi M, Senmaru T, Sakabe K, Tanaka M, Yamazaki M, Hasegawa G, Nakamura N. The coefficient variation of home blood pressure is a novel factor associated with macroalbuminuria in type 2 diabetes mellitus. Hypertens Res 2011; 34: 1271-1275.

5 Schillaci G, Pucci G, Parati G. Blood pressure variability: an additional target for antihypertensive treatment? Hypertension 2001; 58: 133-135.

6 Webb AJ, Fischer U, Mehta Z, Rothwell PM. Effects of antihypertensive-drug class on interindividual variation in blood pressure and risk of stroke: a systematic review and meta-analysis. Lancet 2010; 375: 906-915.

7 Rothwell PM, Howard SC, Dolan E, O'Brien E, Dobson JE, Dahlöf B, Poulter NR, Sever PS. ASCOT-BPLA and MRC Trial Investigators. Effects of beta-blockers and calciumchannel blockers on within-individual variability in blood pressure and risk of stroke. Lancet Neurol 2010; 9: 469-480.

8 Mancia G, Facchetti R, Parati G, Zanchetti A. Visit-to-visit blood pressure variability in the European Lacidipine Study on Atherosclerosis: methodological aspects and effects of antihypertensive treatment. J Hypertens 2012; 30: 1241-1251.

9 Höcht C, Bertera FM, Mayer MA, Taira CA. Issues in drug metabolism of major antihypertensive drugs: beta-blockers, calcium channel antagonists and angiotensin receptor blockers. Expert Opin Drug Metab Toxicol 2010; 6: 199-211.

10 Webb AJ, Fischer U, Rothwell PM. Effects of $\beta$-blocker selectivity on blood pressure variability and stroke: a systematic review. Neurology 2011; 77: 731-737.

11 Su DF, Miao CY. Blood pressure variability and organ damage. Clin Exp Pharmacol Physiol 2001; 28: 709-715.

12 Wang J, Shen FM, Wang MW, Su DF. Effects of nine antihypertensive drugs on blood pressure variability in sinoaortic-denervated rats. Acta Pharmacol Sin 2006; 27 1013-1017.

13 Wang J, Shen FM, Zhang XF, Wang MW, Su DF. Functional arterial baroreflex attenuates the effects of antihypertensive drugs in conscious rats. J Pharmacol Sci 2006; 100: 271-277.

14 Miao CY, Xie HH, Wang JJ, Su DF. Candesartan inhibits sinoaortic denervation-induced cardiovascular hypertrophy in rats. Acta Pharmacol Sin 2002; 23: 713-720.

15 Zhang LC, Li ZZ, Yu YS, Wang ZB, Wei X, Su DF, Li L. Angiotensin-converting enzyme and Angiotensin-converting enzyme 2 are involved in sinoaortic denervation-induced cardiovascular hypertrophy in rats. Biol Pharm Bull 2011; 34: 1398-1403.
16 Krieger EM. Neurogenic hypertension in the rats. Circulation Res 1964; 15: 511-521. 17 Opezzo JA, Höcht C, Taira CA. Pharmacokinetic profile of methyldopa in the brain of sinaortic-denervated rats. Pharmacol Res 2003; 48: 61-67.

18 Opezzo JA, Höcht C, Taira CA, Bramuglia GF. Study of the evolution of blood and striatal levels of methyldopa: a microdialysis study in sinaortic denervated rats. Pharmacol Res 2000; 41: 455-459.

19 Bertera FM, Del Mauro JS, Polizio AH, Chiappetta D, Taira CA, Höcht C. Effect of nebivolol on beat-to-beat and short-term blood pressure variability in spontaneously hypertensive rats. Naunyn Schmiedebergs Arch Pharmacol 2012; 385: 833-843.

20 Bertera FM, Del Mauro JS, Chiappetta D, Polizio AH, Buontempo F, Taira CA, Höcht C. Enantioselective pharmacokinetic and pharmacodynamic properties of carvedilol in spontaneously hypertensive rats: focus on blood pressure variability. Naunyn Schmiedebergs Arch Pharmacol 2012; 385: 325-335.

21 Bertera FM, Mayer MA, Opezzo JA, Taira CA, Höcht C. Comparison of different pharmacodynamic models for PK-PD modeling of verapamil in renovascular hypertension. J Pharmacol Toxicol Methods 2008; 57: 212-219.

22 Di Verniero CA, Bertera F, Buontempo F, Bernabeu E, Chiappetta D, Mayer MA, Bramuglia GF, Taira CA, Höcht C. Enantioselective pharmacokinetic-pharmacodynamic modelling of carvedilol in a N-nitro-l-arginine methyl ester rat model of secondary hypertension. J Pharm Pharmacol 2010; 62: 890-900.

23 Pladys P, Lahaie I, Cambonie G. Thibault G, Lê NL, Abran D, Nuyt AM. Role of brain and peripheral angiotensin $\mathrm{II}$ in hypertension and altered arterial baroreflex programmed during fetal life in rat. Pediatr Res 2004; 55: 1042-1049.

24 Souza HC, Martins-Pinge MC, Dias da Silva VJ, Borghi-Silva A, Gastaldi AC, Blanco JH, Tezini GC. Heart rate and arterial pressure variability in the experimental renovascular hypertension model in rats. Auton Neurosci 2008; 139: 38-45.

25 Irigoyen MC, Cestari IA, Moreira ED, Oshiro MS, Krieger EM. Measurements of renal sympathetic nerve activity in conscious sinoaortic denervated rats. Braz J Med Biol Res 1988; 21: 869-872.

26 Van Vliet BN, Chafe LL. Reduction of blood pressure variability by amlodipine in baroreceptor denervated rats. Clin Exp Hypertens 2000; 22: 645-661.

27 Lacolley P, Bezie Y, Girerd X, Challande P, Benetos A, Boutouyrie P, Ghodsi N, Lucet B, Azoui R, Laurent S. Aortic distensibility and structural changes in sinoaorticdenervated rats. Hypertension 1995; 26: 337-340.

28 Kuroedov A, Cosentino F, Lüscher TF. Pharmacological mechanisms of clinically favorable properties of a selective beta1-adrenoceptor antagonist, nebivolol. Cardiovasc Drug Rev 2004; 22: 155-168.

29 Meier K, Gorbey S, Lemmer B. Effects of nebivolol and metoprolol on nitric oxide urinary excretion, on expression of eNOS and on blood pressure in SH-rats. Naunyn Schmiedebergs Arch Pharmacol 2004; 369 (Suppl 1): R42.

30 Rocha ML, Bendhack LM. Spontaneous oscillatory contractions in aortas of rats with arterial pressure lability caused by sinoaortic denervation. Clin Exp Pharmacol Physiol 2007; 34: 708-713.

31 Zepeda RJ, Castillo R, Rodrigo R, Prieto JC, Aramburu I, Brugere S, Galdames K, Noriega V, Miranda HF. Effect of Carvedilol and Nebivolol on Oxidative Stress-related Parameters and Endothelial Function in Patients with Essential Hypertension. Basic Clin Pharmacol Toxicol 2012; 111: 309-316.

32 Virdis A, Ghiadoni L, Taddei S. Effects of antihypertensive treatment on endothelial function. Curr Hypertens Rep 2011; 13: 276-281.

33 Stauss HM. Identification of blood pressure control mechanisms by power spectral analysis. Clin Exp Pharmacol Physiol 2007; 34: 362-388.

34 Janssen BJ, Oosting J, Slaaf DW, Persson PB, Struijker-Boudier HA. Hemodynamic basis of oscillations in systemic arterial pressure in conscious rats. Am J Physiol 1995; 269: H62-H71.

35 Fazan Jr R, Huber DA, Silva CA, Dias da Silva VJ, Salgado MC, Salgado HC. Sildenafil acts on the central nervous system increasing sympathetic activity. J App/ Physiol 2008; 104: 1683-1689.

36 Just A, Faulhaber J, Ehmke H. Autonomic cardiovascular control in conscious mice. Am J Physiol Regul Integr Comp Physiol 2000; 279: R2214-R2221.

37 Bertera F, Di Verniero CA, Mayer MA, Chiappetta D, Buontempo F, Polizio AH, Taira CA, Höcht C. Pharmacokinetic and pharmacodynamic properties of carvedilol in fructose hypertensive rats. Xenobiotica 2012; 42: 206-219.

38 Chiladakis JA, Georgiopoulou E, Alexopoulos D. Autonomic effects of nebivolol versus atenolol in healthy subjects. Cardiovasc Drugs Ther 2004; 18: 469-473.

39 Sacco G, Evangelista S, Criscuoli M, Goso C, Bigioni M, Binaschi M, Manzini S, Maggi CA. Involvement of nitric oxide in both central and peripheral haemodynamic effect of D/L-nebivolol and its enantiomers in rats. Eur J Pharmacol 2005; 511 : 167-174. 